\title{
Effects of a standardized information booklet on patient anxiety and satisfaction with information at magnetic resonance imaging: a randomized single-blind placebo-controlled trail
}

\author{
Anetta Bolejko ${ }^{1}$ and Peter Hagell ${ }^{2}$ \\ ${ }^{1}$ Lund Univ \\ ${ }^{2}$ Kristianstad University
}

May 5, 2020

\begin{abstract}
Rationale, aims and objectives; Magnetic resonance imaging (MRI) can be perceived as unpleasant even though the examination is noninvasive. Patients' knowledge of the MRI procedure is usually scarce, which might enhance patient anxiety at the examination. The aims of this randomized single-blind placebo-controlled trial were to investigate the effects on anxiety and satisfaction with information of a standardized booklet on MRI compared to a placebo booklet delivered to adult patients prior to their first MRI examination. Method; The intervention group ( $\mathrm{n}=95)$ received a standardized booklet prior to MRI, while the control group $(n=102)$ received a placebo booklet in the same size and lay out but containing general information. The State Trait Anxiety Inventory (STAI) with supplementary questions from the Quality from the Patient's Perspective questionnaire were used as the patient-reported outcome measures. Results; There was no significant difference in anxiety between the groups, either prior to MRI or during the examination, but those who received the placebo booklet were at higher risk of experiencing high anxiety prior to the MRI examination (odds ratio, 2.64; $\mathrm{P}=0.029$ ). The intervention group was more satisfied with the information received $(\mathrm{P}=0.044)$, and a majority of participants in both groups ([?]87\%) considered it important to obtain information on the MRI procedure. Conclusion; Standardized written MRI information decreases the risk of experiencing high anxiety levels before MRI and improves patients' satisfaction with the information. Further research is needed to investigate whether written information prior to MRI is beneficial not only from the patient perspective but if it can also be proven cost-effective.
\end{abstract}

\section{Introduction}

Magnetic resonance imaging (MRI) is an important modality in diagnostics and treatment follow-up. A common non-open MRI camera consists of a tunnel approximately $1.6 \mathrm{~m}$ long and $0.6 \mathrm{~m}$ in diameter surrounded by the magnet ${ }^{1-2}$. The patient lies isolated in the camera during the examination which lasts for approximately 30-60 minutes. The noise level during the examination is high, between 65 and 95 decibels depending on the MRI equipment and procedure, thus ear protection is used during the examination. Although noninvasive, MRI may therefore be perceived as unpleasant and stressful ${ }^{3}$. For example, experiences of fear and a threat of losing self-control have been described during MRI ${ }^{4-5}$, and $14 \%$ of patients experience severe anxiety and fear in MRI ${ }^{6}$. Several studies reported that up to $15 \%$ of patients terminate their MRI due to anxiety and feelings of claustrophobia during the examination ${ }^{7-10}$. Features of MRI equipment and its strange environment have been described as sources of distress ${ }^{4-6}$.

Various measures aiming to alleviate patient discomfort have been investigated ${ }^{11}$; for example, premedication $^{9}$, relaxation exercises ${ }^{12-14}$, hypnosis ${ }^{15}$, a visit prior to $\mathrm{MRI}^{16}$, extensive oral information and counselling ${ }^{14,17}$, and a telephone contact or a video demonstration of the procedure prior to the examination ${ }^{18-19}$. Most of these interventions are time consuming and rather complex to provide in clinical 
practice. Using a questionnaire to identify whether a person has claustrophobia has been suggested ${ }^{20-21}$, and persons with claustrophobia may then be examined in an open magnet ${ }^{22-24}$ or receive premedication ${ }^{9}$. However, open magnets have limitations ${ }^{25}$ and using sedatives may be inconvenient for outpatients due to side effects, and if sedatives are given intravenously, monitoring of the patient also needs to be considered ${ }^{26}$.

Lack of information can worsen anxiety at MRI ${ }^{27-28}$. However, patients' knowledge of how MRI is conducted is usually scarce and the source of information is often relatives ${ }^{29}$, which points to the need for accurate and standardized pre-scanning information. For example, written information before an MRI has been found needed and welcomed by patients ${ }^{3,30}$, and an information booklet would be a simple and inexpensive means to ameliorate the discomfort that might be experienced. It has been stressed that such written patient information should contain several types of information, i.e., procedural (how the examination/treatment is carried out), behavioral (how the patient can cooperate), and sensory (what the patient may experience) information ${ }^{31}$.

However, the evidence regarding the potential effects of a simple intervention such as an information booklet delivered to the patient prior to the MRI examination is scarce. One non-randomized study used written information but in combination with other measures ${ }^{16}$ and found reduced anxiety levels during scanning. Another non-randomized study failed to demonstrate any differences in anxiety or satisfaction with the information between groups receiving standard vs. extended pre-scan written information, although motion artefacts in the MRI images were fewer in the latter group ${ }^{32}$.

The aim of this randomized single-blind placebo-controlled trial was to investigate the effects of a standardized information booklet on patient anxiety and satisfaction with information in connection with MRI. Specifically, the following hypotheses were investigated.

1. A standardized information booklet on MRI decreases patient anxiety prior to and during MRI scanning.

2. A standardized information booklet on MRI increases patient satisfaction with information about the procedure.

\section{Methods}

The study was conducted in accordance with the Declaration of Helsinki and was reviewed by the local ethics advisory committee. All participants gave their informed consent.

\section{Design}

This is a placebo-controlled single-blind randomized controlled trial on the effects of standardized written information in comparison with a placebo intervention on patient anxiety and satisfaction with information among adults undergoing an MRI examination for the first time.

Sample

Study participants were consecutively recruited during 18 months at the Department of Medical Imaging and Physiology at University Hospital in Malmö, Sweden. The department has 4 MRIs and performs approximately 5000 outpatient MRI examinations each year. The inclusion criteria were outpatients aged 18-70 years who were to undergo an MRI examination using a non-open camera Siemens Symphony 1.5 Tesla MRI scanner. Inpatients, those who previously had undergone an MRI examination, those who were to undergo MRI in anesthesia, patients with cognitive impairment, and those who did not speak Swedish were excluded. The study participants were randomized to either an intervention or control group. The flow chart of the study sample is presented in Figure 1.

Figure 1 about here

The sample size was estimated a priori based on previous study results regarding anxiety, as assessed using the State Trait Anxiety Inventory (STAI); pre-scanning mean scores in the intervention and control groups were estimated to be 37 and 41, respectively, and scores after MRI were estimated to be 32 and 37, respectively, 
with a common SD of 10. Based on these parameters, which give effect sizes of 0.4 and 0.5 , respectively, approximately 99 and 64 individuals were required per group for $80 \%$ power at a two-tailed alpha level of $0.05^{33}$.

Intervention

The active intervention consisted of an information booklet on MRI that was developed based on clinical experience, relevant literature, and patient input ${ }^{31}$. Briefly, the booklet contained the following information:

- explanation of how an MRT examination is conducted (procedure information)

- description of what might be expected by the patient in connection to the MRI procedure (behavior information)

- examples of potential reactions and experiences in connection to MRT (sensory information)

The placebo intervention consisted of an information booklet of the same size and layout as the intervention booklet, but only containing general information about the department and no specific information about the MRI procedure.

In addition to the booklets, both groups received standard care. This consists of checking responses to a safety questionnaire that patients complete before the appointment. This is followed by oral information about the examination (e.g., duration of the examination, the need to lie still, and that a loud noise will be heard when images are taken). If the procedure requires the patient to hold her/his breath, this is practiced prior to the examination. Ear protection is applied and those who wish so are offered to listen to music. Everyone gets an alarm clock to call on the staff if needed. Relatives are welcome to be present in the examination room but have no opportunity to communicate with the patient. The time to give the above information is limited to approximately 5 minutes. Upon completion of the MRI, the patient is informed that the results will be sent to the referring physician.

Instruments and other study data

Anxiety was assessed using the STAI, which consists of two parts: STAI FORM Y-2 T and STAI FORM Y-1 $\mathrm{S}^{34}$. STAI FORM Y-2 T ("trait anxiety"), assesses the individual's general tendency to experience stressful situations as worrying, and STAI FORM Y-1 S ("state anxiety") assesses the current anxiety levels (ibid). STAI FORM Y-1 S is available in two different designs; one is worded in present tense and intends to assess the respondent's anxiety right now and the other is worded in past tense and intends to assess recently perceived anxiety. These STAI forms are hereinafter referred to as STAI-T (trait anxiety), STAI-SB and STAI-SA (state anxiety before and immediately after MRI, respectively, where the STAI-SA was intended to assess anxiety experienced during the MRI). All STAI forms comprise 20 items each with four ordered response categories scored 1-4, yielding possible total scores between 20 (less anxiety) and 80 (more anxiety). A total score of 40 or higher is considered to indicate a high level of anxiety ${ }^{16}, 32$. In cases of [?]10\% missing item responses, scores are imputed by averaging item scores across available item responses ${ }^{34}$.

The STAI has been used in a variety of areas, including the assessment of anxiety in the context of MRI scanning $12-13,16-17,19,23-24,32,35$. There is general support for the validity and reliability of scores on the different STAI forms ${ }^{36}$. In the present study, the Swedish version ${ }^{37}$ of the instrument was used. Cronbach's coefficient alpha in the control and intervention groups in this study was 0.94 and 0.92 for STAI-T, 0.93 and 0.94 for STAI-SB, and 0.94 and 0.93 for STAI-SA, respectively.

Satisfaction with the information about MRI was assessed using three study specific items. The first question concerned the agreement between the patient's expectations of the examination and the actual experience. The two other questions were adapted from the Quality from the Patient's Perspective questionnaire for mammography ${ }^{38}$ and concerned whether the pre-scan information made them understand what was going to happen, and how important the patient considers such information to be. All three questions had four ordered response categories scored 1-4, where 4 represents a higher degree of satisfaction. 
In addition, it was recorded whether or not sedatives were taken prior to the MRI examination, relatives were present in the examination room, and if the patient listened to music during the examination. Other data recorded were age, gender, duration of the examination, scanned body part, if the patient called or visited the MRI department before the examination, if the patient aborted the examination, and medical history (classified as malignant or other disease) at referral.

Data collection

An invitation to participate was sent together with the call letter for the MRI examination. Those who wished to participate were asked to sign and return a written informed consent together with the completed STAI-T. To mimic routine clinical use of the written MRI information as far as possible, those randomized to the intervention group received the MRI information booklet together with the call letter and the control group received the placebo booklet.

All staff at the MRI unit were fully informed about the study but were blinded to which group patients belonged to. Prior to MRI, all patients were asked to complete the STAI-SB and sealed it in an envelope, before being provided standard care (see above). Immediately after completion of the MRI, patients were asked to complete the STAI-SA as well as the three satisfaction questions, which also were returned in sealed envelopes. Patients who aborted the examination prematurely were also asked if they were willing to answer the questionnaires.

Data analysis

Data were analyzed using IBM SPSS Statistics 25, with the two-tailed significance level set to $\mathrm{P}<0.05$. Demographic data, intake of sedatives, the presence of relatives in the MRI room, whether the patients listened to music in connection to the examination, STAI-T scores, and other baseline data were analyzed using chi-square/Fisher's exact tests, Mann-Whitney U tests and independent t-tests, as appropriate. MannWhitney U and Wilcoxon signed-rank tests were used for between and within group comparisons of STAI-SB and STAI-SA scores, and Mann-Whitney U tests were used to compare satisfaction scores.

The proportion of patients with STAI-SB and STAI-SA total scores of 40 or more was compared between the intervention and control groups using chi-square tests. To examine whether the intervention booklet was associated with a lower risk of high anxiety immediately before and during MRI scanning, a multivariate logistic regression (forward stepwise) was performed, using dichotomized STAI-SB and STAI-SA total scores $(<40=0 ;[?] 40=1)$ as dependent variables. Group assignment (intervention or control), high trait anxiety (STAI-T [?]40), gender, telephone contact or visiting MRI prior to the examination, use of sedatives prior to MRI, presence of relatives in the examination room, music during the examination, and medical history were entered as independent variables.

\section{Results}

The study sample consisted of 109 (55\%) women and 88 (45\%) men. Other demographic data is presented in Table 1. Five people in the intervention group but none in the control group visited the MRI unit prior to their scheduled examination. No other group differences were found regarding demographic and other sample characteristics, including general tendency to experience anxiety (STAI-T scores).

Table 1 about here

Anxiety prior to and during MRI

There was no significant difference in anxiety between the intervention and control group, either prior to MRI or during the examination (Table 2). Both groups showed significantly lower anxiety during MRI compared to immediately before the examination. Group comparisons were also conducted excluding the patients in the intervention group who visited the MRI unit prior to the examination. The analysis yielded median $\left(\mathrm{q}_{1}-\mathrm{q}_{3}\right)$ STAI-SB $(\mathrm{n}=79)$ and STAI-SA $(\mathrm{n}=75)$ scores of 32 (25-39) and 27 (22-37) for the intervention group. Compared to the control group (Table 2), these results gave P-values of 0.231 and 0.421 for the STAI-SB and STAI-SA, respectively. 


\section{Table 2 about here}

Thirty-three respondents $(39 \%)$ in the control group and $22(25 \%)$ in the intervention group had a total STAI-SB score [?]40 ( $\mathrm{P}=0.124$; chi-square test). For the STAI-SA, equal numbers of respondents in both groups $(\mathrm{n}=17)$ had a total score [?]40 ( $\mathrm{P}=0.843$; chi-square test). Logistic regression demonstrated that high trait anxiety (total STAI-T scores [?]40), having visited the MRI unit prior to the examination, presence of relatives at in the examination room, and receiving the placebo intervention rather than the MRI information booklet were associated with high levels of pre-scan anxiety (Table 3). High anxiety levels during scanning (STAI-SA scores [?]40) were associated with high trait anxiety and having called the MRI unit prior to the examination (Table 3 ).

Table 3 about here

\section{Satisfaction with information}

Participants in both groups found pre-scan information to be of importance (median [q1-q3], 4 [3-4] in both groups; $\mathrm{P}=0.837$; Mann-Whitney U-test). Both groups also considered their expectations of the examination to agree with their experiences (median [q1-q3], 3 [2-4] and 3 [3-4] for the control and intervention groups, respectively; $\mathrm{P}=0.253$; Mann-Whitney U-test). However, the intervention group was more satisfied with the given information (median [q1-q3], 4 [4-4]) than the control group was (4 [3-4]; P=0.044; Mann-Whitney U-test).

\section{Discussion}

In this randomized single-blind placebo-controlled trial the effects of a standardized MRI information booklet on anxiety and satisfaction with information among adult outpatients undergoing their first MRI examination was investigated. There were no differences in anxiety between the intervention and control groups before or during scanning, but those who received the placebo booklet had a significantly higher risk of experiencing severe anxiety immediately prior to MRI. The results also showed that pre-scanning information is considered important, and that those who received MRI specific written information before their examination were more satisfied than those who received general information.

The effects of written patient information on elevating patient anxiety associated with MRI have been investigated before using the STAI questionnaire 16, 32. One study found significantly decreased anxiety during MRI among patients who received an information booklet in comparison with those who did not 16. However, the written information in that study was supplemented by other interventions such as a prescan counselling, which makes it uncertain to what extent the outcomes can be attributed to the written informationper se. Another study failed to demonstrate any reduction in MRI-associated anxiety from provision of an information booklet alone ${ }^{32}$. This may have been due to providing written MRI information to both study groups and only supplemental information to the experimental group ${ }^{32}$. However, the present study demonstrates that those who received the placebo booklet had a higher risk of experiencing high prescan anxiety compared to those who received specific MRI information. This association was independent and beyond those of high baseline anxiety levels, pre-scan visits to the MRI and the presence of relatives during scanning. Furthermore, factors such as cause of referral, use of sedatives and gender, which all have been found associated with MRI anxiety in previous studies ${ }^{9}, 19,32$, did not contribute to high anxiety levels once the written information was taken into account. These findings are strengthened by the randomized placebo-controlled design, which differs from previous quasi-experimental studies of the effects of written information on MRI associated anxiety 16,32 .

The comparison within the groups regarding patient perceived anxiety prior to and during the MRI showed significant differences for both groups. This may be due to a feeling of relief after the examination. That is, although STAI-SA intended to assess anxiety during the examination, it cannot be ruled out that responses may have been influenced by perceptions at the time when the inventory was answered. Furthermore, attendance and oral information by the staff (which was provided after the STAI-SB was responded to) may also have had an anxiety reducing effect ${ }^{4-5,39}$. For example, it has been found that support from the staff 
have a significant impact on patients ${ }^{6}$ experiences of an MRI and the staff/patient interaction may facilitate self-control and coping during the examination ${ }^{4}$.

The intervention group was more satisfied with the information compared to the control group, which is contrary to previous results in $\mathrm{MRI}^{32}$. This could be explained that our study provided a booklet that was developed in interaction with people who had undergone an MRI, which probably enhances the relevance and comprehension of the information ${ }^{31}$.

A placebo booklet was applied, which is considered a strength of the study. Nevertheless, there is also a risk to use placebo considering that the attention provided by the placebo booklet may have affected their experiences of MRI, even though it did not include any information about the examination. However, it has been argued that these effects are negligible in interventions of patient education that are applied over a short period of time ${ }^{40}$. Indeed, the Nordic Cochrane Centre concluded that in general there is no evidence that placebo interventions have clinical effect with an exception of possible influence on experiences of pain and nausea ${ }^{41}$.

One aspect that always should be considered in relation to any intervention is its cost-effectiveness. That is, whether the extra cost associated with printing and distributing an MRI information booklet is compensated by its effects. To the best of our knowledge, there is no such evidence available regarding written information prior to MRI. However, it appears reasonable to consider the relatively small costs to be acceptable in view of the results presented here, particularly since previous studies have suggested that this type of intervention also may reduce motion artefacts ${ }^{32,42}$. Nevertheless, future studies should consider cost-effectiveness in addition to motion artefacts and MRI associated anxiety and wellbeing ${ }^{43}$.

Conclusion

This placebo-controlled single-blind randomized trial examined the effects of standardized written information to people undergoing their first MRI examination. The results showed that such information reduces the risk of experiencing high pre-scanning anxiety levels, that patients find it important to receive information before the examination, and that it increases the satisfaction with the information as compared to general information.

\section{References}

1. Westbrook C, Kaut Roth C, Talbot J. MRI in Practice. Oxford: Wiley-Blackwell; 2011.

2. Siemens Healthcare. Siemens Healthineers. Magnetic Resonance Imaginghttps://www.siemenshealthineers.com/magnetic-resonance-imaging 2019

3. Munn Z, Jordan Z. The patient experience of high technology medical imaging: A systematic review of the qualitative evidence. Radiography 2011;17:323-331.

4. Tornqvist E, Mansson A, Larsson E-M, Hallstrom, I. It's like being in another world. Patients' lived experience of magnetic resonance imaging. J Clin Nurs 2006;15:954-961.

5. Carlsson S, Carlsson E. The situation and the uncertainty about the coming result scared me but interaction with the radiographers helped me through: a qualitative study on patients' experiences of magnetic resonance imaging examinations. J Clin Nurs 2013;22:3225-3234.

6. Katznelson R, Djaiani GN, Minkovich L, Fedorko L, Carroll J, Borger MA, Cusimano RJ, Karski J. Prevalence of claustrophobia and magnetic resonance imaging after coronary artery bypass graft surgery. Neuropsychiatr Dis Treat 2008;4:487-493.

7. Kilborn LC, Labbe EE. Magnetic resonance imaging scanning procedures: Development of phobic response during scan and at on-month follow-up. Int J Behav Med 1990;13:391-401.

8. Melendez JC, McCrank E. Anxiety-related reactions associated with magnetic resonance imaging examinations. JAMA 1993;270:745-747.

9. Hollenhorst J, Munte S, Friedrich L, HeineJ, Leuwer M, Becker H, Piepenbrock S. Using intranasal midazolam spray for prevent claustrophobia induced by MR imaging. AJR 2001;176:864-868.

10. Eshed I, Althoff CE, Hamm B, Hermann K-G. Claustrophobia and premature termination of magnetic resonance imaging examinations. J Magn Reson Imaging 2007;26:401-404. 
11. Munn Z, Jordan Z. Interventions to reduce anxiety, distress and the need for sedation in adult patients undergoing magnetic resonance imaging: a systematic review. Int J Evid Based Healthc 2013;11:265274 .

12. Thompson MB, Coppens NM. The effects of guided imagery on anxiety levels and movement of clients undergoing magnetic resonance imaging. Holist Nurs Pract 1994;8:59-69.

13. Lukins R, Davan IGP, Drummond PD. A cognitive behavioural approach to preventing anxiety during magnetic resonance imaging. J Behav Ther Exp Psychiatry 1997;28:97-104.

14. Lang EV, Ward C, Laser E. Effect of team training on patients' ability to complete MRI examinations. Acad Radiol 2010;17:18-23.

15. Friday PJ, Kubal WS. Magnetic resonance imaging: improved patient tolerance utilizing medical hypnosis. Am J Clin Hypn 1990;33:80-84.

16. Grey SJ, Price G, Mathews A. Reduction of anxiety during MR imaging: a controlled trial. Magn Reson Imaging 2000;18:351-355.

17. Tazegul G, Etcioglua E, Yildiza F, Yildiza R, Tuney T. Can MRI related patient anxiety be prevented? Magn Reson Imaging 2015;33:180-183.

18. Ahlander B-M, Engvall J, Maret E, Ericsson E. Positive effect on patient experience of video information given prior to cardiovascular magnetic resonance imaging: A clinical trial. J Clin Nurs 2018;27:1250-1261.

19. Tugwell RJ, Goulden N, Mullinsc P. Alleviating anxiety in patients prior to MRI: A pilot single-centre single-blinded randomised controlled trial to compare video demonstration or telephone conversation with a radiographer versus routine intervention. Radiography 2018;24:122-129.

20. McIsaac HK, Thordarson DS, Shafran R, Rachman S, Poole G. Claustrophobia and the magnetic resonance imaging procedure. J Behav Med 1998;21: 255-268.

21. Radomsky AS, Rachman S, Thordarson DS, MaIsaac HK, Teachman BA. The claustrophobia questionnaire. J Anxiety Disord 2001;15:287-297.

22. Spouse E, Gedroyc WM. MRI of the claustrophobic patient: interventionally configured magnets. The British Journal of Radiology 2000;73:146-151.

23. Bangard C, Paszek J, Berg, Eyl G, Kessler J, Lackner K, GossmanA. MR imaging of claustrophobic patients in an open $1.0 \mathrm{~T}$ scanner: Motion artifacts and patient acceptability compared with closed bore magnets. Eur J Radiol 2007;64:152-157.

24. Enders J, Zimmermann E, Rief M, Martus P, Klingebiel R, Asbach P, Klessen C, Diederichs G, Bengner T, Teichgraber U, Hamm B, Dewey M. Reduction of claustrophobia during magnetic resonance imaging: methods and design of the "CLAUSTRO" randomized controlled trial. BMC Med Imaging 2011;11:4.

25. Lederer W. Practical suggestions for improving MRI throughput and quality. Radiol Manage 1994;16:32-35.

26. Murphy KJ, Brunberg JA. Adult claustrophobia, anxiety and sedation in MRI. Magn Reson Imaging 1997;15:51-54.

27. Katz RC, Wilson L, Franzer N. Anxiety and its determinations in patients undergoing magnetic resonance imaging. J Behav Ther Exp Psychiatry 1994;25:131-134.

28. Chandler T. Techniques for optimizing MRI. Relaxation and visualization. Adm Radiol J 1996;3:16-18.

29. Chesson RA, McKenzie GA, Mathers SA. What do patients know about ultrasound, CT and MRI? Clin Radiol 2002;57:477-482.

30. Mitchell M. Constructing information booklets for daycase patients. Ambulatory Surgery 2001;9;37-45.

31. Bolejko A., Sarvik C, Hagell P, Brinck A. Meeting patient information needs prior to magnetic resonance imaging: development and evaluation of an information booklet. J Radiol Nurs 2006;27:96-102.

32. Tornqvist E, Mansson A, Larsson E-M, Hallstrom I. Impact of extended written information on patients'anxiety and image motion artefacts at magnetic resonance imaging. Acta Radiol 2006;47:474-480.

33. Cohen J. Statistical Power Analysis for the Behavioural Sciences. Mahwah: Lawrence Erlbaum Associates, Inc; 1988.

34. Spielberger CD. Manual for the State-Trait Anxiety Inventory. STAI (Form Y). Self-evaluation questionnaire. Redwood City California: Mind Garden Inc.; 1983. 
35. Selim MA. Effects of pre-instruction on anxiety levels of patients undergoing magnetic resonance imaging examination. East Mediterr Health J 2001;7:519-525.

36. McDowell I. Measuring health: a guide to rating scales and questionnaires. Third edition. New York: Oxford University Press, Inc.; 2006.

37. Forsberg C, Bjorvell H. Swedish population norms for the GHRI, HI and STAI-state. Qual Life Res 1993;2:349-356.

38. Wilde Larsson B, Larsson G, Larsson M, Starrin B. KUPP-boken. Vagledning till frageformularet KUPP, kvalitet ur patientens perspektiv. Stockholm: Grafiska Gruppen; 2001.

39. Nazemi H, Dager SR. Coping strategies of panic and control subjects undergoing lactade infusion during magnetic resonance imaging confinement. Comprehensive Psychiatry 2003;44:190-197.

40. Chan CWD, Thompson DR. The use of placebo in nursing research. Journal of Clinical Nursing 2006;15:521-524.

41. Hrobjartsson A, Gotzsche PC. Placebo interventions for all clinical conditions. Cochrane Database Syst Rev 2010;20:CD003974.

42. Ahlander B-M, Arestedt K, Engvall J, Maret E, Ericsson E. Development and validation of a questionnaire evaluating patient anxiety during Magnetic Resonance Imaging: the Magnetic Resonance Imaging-Anxiety Questionnaire (MRI-AQ). JAN 2016;72:1368-1380.

43. Ali HH, ModicME, Mahmoud SY, Jones SE. Reducing clinical MRI motion degradation using a prescan patient information pamphlet. AJR 2013;200: 630-634.

44. Barlett EE. Cost-benefit analysis of patient education. Patien Education and Counseling 1995;26:87-91.

\section{Acknowledgments}

The authors would like to thank the Department of Medical Imaging and Physiology in Malmo for the possibility to conduct the study. We are also grateful to the staff at the department for their help in collecting patient self-report questionnaires.

\section{Conflicts of interest statement}

No conflict of interest

Table 1. Demographic data and other characteristics of the study groups.

\begin{tabular}{|c|c|c|c|}
\hline & Intervention group $\mathrm{n}=95$ & Control group $n=102$ & $\mathrm{P}$-value \\
\hline $\begin{array}{l}\text { Gender, man/woman, } \\
\mathrm{n}(\%)\end{array}$ & $43(45) / 52(55)$ & $45(44) / 57(56)$ & $0.872^{+}$ \\
\hline Age (years), mean (SD) & $48.7(12.9)$ & $49.4(13.0)$ & $0.686^{++}$ \\
\hline $\begin{array}{l}\text { Duration of MRI } \\
\text { (minutes), mean (SD) }\end{array}$ & $34.0(13.1)$ & $34.3(16.7)$ & $0.857^{++}$ \\
\hline Aborted MRI, n (\%) & $2(2.1)$ & $4(3.9)$ & $0.684^{\S}$ \\
\hline $\begin{array}{l}\text { Telephone call prior to } \\
\text { MRI, n (\%) }\end{array}$ & $13(13.7)$ & $7(6.9)$ & $0.113^{+}$ \\
\hline $\begin{array}{l}\text { Visit prior to MRI, n } \\
(\%)\end{array}$ & $5(5.3)$ & $0(0)$ & $0.025^{\S}$ \\
\hline $\begin{array}{l}\text { Sedatives prior MRI, } \mathrm{n} \\
(\%)\end{array}$ & $5(5.3)$ & $8(7.8)$ & $0.476^{+}$ \\
\hline $\begin{array}{l}\text { Relatives present in the } \\
\text { MRI room, } \mathrm{n}(\%)\end{array}$ & $10(10.5)$ & $13(12.7)$ & $0.609^{+}$ \\
\hline $\begin{array}{l}\text { Music during MRI, n } \\
(\%)\end{array}$ & $58(62.1)$ & $63(61.8)$ & $0.939^{+}$ \\
\hline $\begin{array}{l}\text { Referral diagnosis, } \\
\text { malignancy/other, } \mathrm{n} \\
(\%)\end{array}$ & $28(29.5) / 67(70.5)$ & $29(28.4) / 73(71.6)$ & $0.872^{+}$ \\
\hline
\end{tabular}




\begin{tabular}{|c|c|c|c|}
\hline & Intervention group $\mathrm{n}=95$ & Control group $n=102$ & $\mathrm{P}$-value \\
\hline $\begin{array}{l}\text { Trait anxiety } \\
(\text { STAI-T }), \text { median }\left(q_{1}\right. \\
\left.-q_{3}\right)\end{array}$ & $33(28-43)$ & $33(27-44)$ & $0.850^{\mathrm{PP}}$ \\
\hline $\begin{array}{l}\text { Investigated bodypart, } \mathrm{n} \\
\text { (\%) Head } \\
\text { Thorax/abdomen Spine } \\
\text { Upper extremity Lower } \\
\text { extremity }\end{array}$ & $\begin{array}{l}38(40.0) 25(26.3) 16 \\
(16.8) 4(4.2) 12(12.6)\end{array}$ & $\begin{array}{l}31(30.4) 24(23.5) 24 \\
(23.5) 1(1.0) 22(21.6)\end{array}$ & $0.154^{\S}$ \\
\hline
\end{tabular}

${ }^{+}$Chi-square test; ${ }^{++}$T-test; ${ }^{\mathrm{SS}}$ Fisher's exact test; ${ }^{\mathrm{PP}}$ Mann-Whitney U-test MRI, magnetic resonance imaging; SD, standard deviation; $\mathrm{q}_{1}-\mathrm{q}_{3}, 25^{\text {th }}$ to $75^{\text {th }}$ percentiles

Table 2. State anxiety before and after MRI as compared between and within groups.

\begin{tabular}{llll}
\hline & STAI-SB & STAI-SA & P-value ${ }^{++}$ \\
\hline $\begin{array}{l}\text { Intervention group, } \mathrm{n} \\
\text { median (q1-q3) }\end{array}$ & $\mathrm{n}=8432.8(26.0-41.0)$ & $\mathrm{n}=8027.5(22.0-37.5)$ & 0.002 \\
$\begin{array}{l}\text { Control group, n median } \\
\left(\mathrm{q}_{1} \text {-q }_{3}\right)\end{array}$ & $\mathrm{n}=8935.0(27.0-42.6)$ & $\mathrm{n}=8530.0(21.0-37.4)$ & $<0.001$ \\
$\mathrm{P}_{\text {-value }}{ }^{+}$ & 0.437 & 0.694 & \\
\hline
\end{tabular}

+ Mann-Whitney U-test

++ Wilcoxon test

MRI, magnetic resonance imaging; STAI-SB, state anxiety before scanning; STAI-SA, state anxiety during scanning; $\mathrm{q}_{1}-\mathrm{q}_{3}, 25^{\text {th }}$ to $75^{\text {th }}$ percentiles

Table 3. Multivariate logistic model ${ }^{+}$of predictors for high anxiety levels ([?]40) prior to (STAI-SB) and during (STAI-SA) MRI examination.

\begin{tabular}{lllll}
\hline Anxiety $^{++}$ & Significant predictors $\S$ & B (SE) & P-value & Odds ratio (95\% CI) \\
STAI-SB & High trait anxiety (STAI-T) & $2.36(0.44)$ & $<0.001$ & $10.64(4.45,25.40)$ \\
& Visit prior to MRI & $3.44(1.28)$ & 0.007 & $31.06(2.55,378.88)$ \\
& Relatives present in the MRI room & $1.46(0.59)$ & 0.013 & $4.31(1.36,13.69)$ \\
\multirow{2}{*}{ STAI-SA } & Placebo intervention & $0.97(0.45)$ & 0.029 & $2.64(1.10,6.33)$ \\
& High trait anxiety (STAI-T) & $1.26(0.42)$ & 0.003 & $3.54(1.55,8.05)$ \\
& Telephone call prior to MRI & $1.27(0.42)$ & 0.023 & $3.57(1.19,10.70)$ \\
\hline
\end{tabular}

+ Forward stepwise (likelihood-ratio) multivariate logistic regression

++ Dichotomized total score according to the cut-off value $(<40=0 ;[?] 40=1)$

$\S$ Independent variables entered into the model: gender $(\operatorname{man}=0$, woman $=1)$, visit prior to MRI $($ No $=$ 0 , Yes $=1)$, telephone call prior to MRI $(\mathrm{No}=0$, Yes $=1)$, sedatives prior to MRI $($ No $=0$, Yes $=1)$, relatives present in the MRI room $(\mathrm{No}=0$, Yes $=1)$, music during MRI $(\mathrm{No}=0$, Yes $=1)$, referral diagnosis (malignancy $=1$, other disease $=0)$, high trait anxiety (STAI-T total score $<40=0$, STAI-T total score $[?] 40=1$ ), group affiliation (intervention group $=0$, control group $=1$ ). Independent variables that are not presented in the table were not significantly associated with experiencing high anxiety levels. 
Hosmer-Lemeshow goodness-of-fit test, $\mathrm{P}=0.601$; Nagelkerke‘s pseudo R-square, 0.399 (STAI-SB);

Hosmer-Lemeshow goodness-of-fit test, $\mathrm{P}=0.371$; Nagelkerke‘s pseudo R-square, 0.149 (STAI-SA).

B, regression coefficient; SE, standard error; 95\% CI, 95\% confidence interval; MRI, magnetic resonance imaging; STAI-SB, state anxiety before scanning; STAI-SA, state anxiety during scanning

\section{Figure legends}

Figure 1: Flow chart of the study sample.

\section{Hosted file}

Figure 1.docx available at https://authorea.com/users/302269/articles/432298-effects-of-astandardized-information-booklet-on-patient-anxiety-and-satisfaction-with-informationat-magnetic-resonance-imaging-a-randomized-single-blind-placebo-controlled-trail 